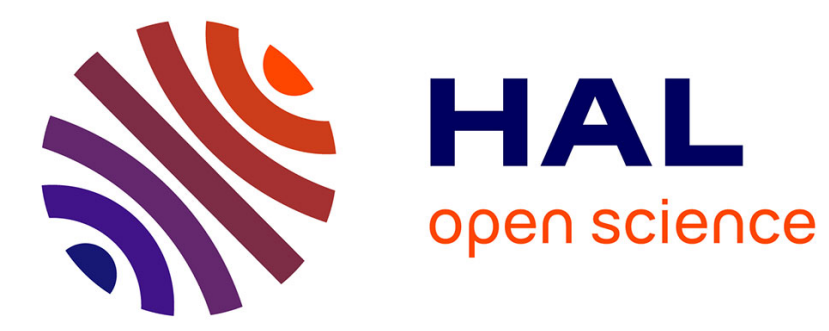

\title{
Un exemple d'estimation de la demande de transport urbain
}

\author{
Stéphanie Souche
}

\section{To cite this version:}

Stéphanie Souche. Un exemple d'estimation de la demande de transport urbain. Revue d'économie régionale et urbaine, 2009, 4, pp. 759-779. 10.3917/reru.094.0759 . halshs-00579638

\section{HAL Id: halshs-00579638 \\ https://shs.hal.science/halshs-00579638}

Submitted on 28 Mar 2011

HAL is a multi-disciplinary open access archive for the deposit and dissemination of scientific research documents, whether they are published or not. The documents may come from teaching and research institutions in France or abroad, or from public or private research centers.
L'archive ouverte pluridisciplinaire HAL, est destinée au dépôt et à la diffusion de documents scientifiques de niveau recherche, publiés ou non, émanant des établissements d'enseignement et de recherche français ou étrangers, des laboratoires publics ou privés. 
Un exemple d'estimation de la demande de transport urbain

Souche S.

Revue d'Économie Régionale \& Urbaine, 4 (2009) pp. 759-779 - http://halshs.archivesouvertes.fr/halshs-00579638/fr/

DOI : $10.3917 /$ reru.094.0759

\title{
Un exemple d'estimation de la demande de transport urbain
}

\section{An example in urban transport demand estimation}

\author{
Par Stéphanie Souche \\ Maître de Conférences, Université Blaise Pascal, Clermont-Ferrand II \\ Laboratoire d'Economie des Transports \\ (CNRS-Université Lyon 2-ENTPE) \\ ISH, 14 av Berthelot \\ 69363 Lyon (FRANCE) \\ Téléphone : +33 472726457 Fax : +33 472726448 \\ Courriel : stephanie.souche@let.ish-lyon.cnrs.fr
}

Mots-clés : Chine, mobilité urbaine, estimation de la demande, long terme, densité urbaine

Key-words : China, urban mobility, demand estimation, long term, urban density

Classification JEL : R0, R10, R40, R41 


\section{Résumé}

Pour anticiper au mieux les villes de demain, un travail de prospective sur la demande de transport urbain est nécessaire. Pour y répondre, l'étude calibre un modèle de demande de mobilité urbaine construit sur les principales variables structurelles identifiées dans la littérature. Ce modèle est appliqué à trois villes chinoises pour lesquelles ont été construits des scénarios d'évolution. Les résultats montrent que deux variables se démarquent: le coût d'usage des déplacements - en voiture particulière et en transports en commun - et la densité urbaine. De manière surprenante mais qui s'explique par les données disponibles, les fonctions de demande estimées s'avèrent indépendantes du groupe de pays d'appartenance. Néanmoins, l'application au cas chinois nous fournit un premier résultat empirique.

\section{Summary}

In order to better understand tomorrow's cities, we need to work on urban transport demand estimation. In this paper, we aim at defining an urban mobility demand model built on the main structural variables currently identified in the literature. By applying it to three Chinese cities, we obtain different evolution scenarios that underline the dominance of the two following variables : average user cost for a trip (in individual cars or public transport) and urban density. Surprisingly enough, the demand estimation functions appear to be independant from the country group variable, though this may be explained by the limited data available. Nevertheless, this application to the Chinese environnement gives us a first empirical result to work on.

\section{$-1-$ \\ Introduction générale ${ }^{1}$}

Il existe une demande sociale forte pour disposer dès aujourd'hui de données qui permettent d'anticiper et de préparer au mieux l'avenir. Les transports n'échappent pas à la règle. Disposer par exemple d'ordre de grandeurs sur la mobilité urbaine des personnes fournit des éléments pour comprendre à quoi pourront ressembler les villes de demain. L'une des difficultés consiste à utiliser le modèle le mieux adapté pour faire de la prospective. C'est à cet exercice que nous voulons nous livrer.

A travers des projections de trafic sur l'ensemble des modes de transport, Schafer et Victor (2000) ont estimé que la mobilité des pays à économie planifiée d'Asie (Vietnam, Mongolie, Chine) représenterait 10,3\% de la mobilité mondiale en 2050 (contre 3,5\% en 1990). La Chine occuperait alors une position dominante dans ce groupe. Par ailleurs, les conclusions du rapport B.R.I.C. (Wilson et Purushothaman, 2003) estiment que la Chine aura atteint en 2050 le PIB du Japon aujourd'hui.

Cette perspective de forte croissance de la mobilité chinoise nous conduit à nous intéresser à l'estimation de sa demande de transport. Elle nous fournit l'occasion d'appliquer le modèle d'estimation de la demande que nous proposons.

L'estimation de la demande de transport à long terme requiert une modélisation spécifique qui identifie les facteurs structurels de mobilité. L'un des premiers facteurs identifiés est la quantité de bien ou de service disponibles, qu'il s'agisse de voiture particulière (Mogridge, 1967 et 1989 ; Evans, 1970 ; Jansson, 1989 ; Button et al., 1993 ; Gakenheimer, 1999 ; Ortuzar et Willumsen, 2006 ; Holmgren, 2007) ou de transports en commun (Bresson et al., 2003, 2004). Mais on retrouve aussi d'autres facteurs tels que le coût d'usage d'un déplacement (Mc Fadden, 1974), le revenu du ménage amené à se déplacer (Schafer et Victor, 2000 ; Dargay et Hanly, 
2002 ; Medlock et Soligo, 2002) ou la manière dont les transports sont intégrés dans l'espace (Kain et Fauth, 1977 ; Oum et al., 1992 ; Giuliano et Dargay, 2006 ; Davidson et al., 2007).

Nous allons construire un modèle de la demande qui s'appuie sur ces différents facteurs structurels. Nous vérifierons s'ils sont statistiquement significatifs et s'ils interviennent de manière équivalente sur la mobilité urbaine. Nous appliquons ensuite ce modèle au cas de trois villes chinoises pour lesquelles nous allons construire différents scénarios d'évolution de la mobilité à horizon 2050.

Nos résultats montrent que deux variables se démarquent des autres : le coût d'usage des déplacements (en voiture particulière et en transports en commun) et la densité urbaine. Ces variables explicatives résistent le mieux aux différents tests économétriques. La valeur des coefficients d'élasticité s'avère par ailleurs conforme à celle que l'on peut trouver dans la littérature. Les fonctions de demande estimées semblent, en outre, indépendantes du groupe de pays d'appartenance. Ceci peut sembler surprenant mais s'explique par l'impossibilité de pouvoir prendre correctement en compte la variable de PIB urbain. Enfin, l'application de notre modèle au cas chinois nous fournit une première estimation empirique de la mobilité urbaine.

Après une revue de littérature sur les facteurs structurels explicatifs de la demande de transport urbain (section 2), nous présenterons le modèle retenu pour estimer la demande de transport (section 3). Nous poursuivrons en exposant et en discutant les résultats des estimations puis nous les appliquerons au cas chinois (section 4).

\section{- 2 -}

\section{Une revue de la littérature sur l'estimation de la demande}

La littérature sur l'estimation de la demande de transport urbain est abondante. Deux méthodes sont couramment utilisées.

La première s’appuie sur le modèle de transport à quatre étapes. Ce type de modèle a besoin de nombreuses données, le plus souvent désagrégées (matrice origines-destinations, coûts généralisés, facteur d'attraction, en emplois notamment, des différentes zones) qui dans le cas chinois sont soit absentes (Boyce, 2006) soit peu fiables (Huenemann, 2001).

La seconde méthode consiste à identifier les variables explicatives de la demande de transport. C'est cette technique qui est le plus souvent utilisée pour estimer la demande à long terme. Elle a besoin de données agrégées et recherche les variables explicatives structurelles de la demande. Ces variables peuvent s'organiser en quatre grands effets: l'effet prix, l'effet revenu, l'effet quantité et l'effet spatial.

\subsection{L'effet prix}

Mc Fadden (1974) met en évidence un effet prix sur la demande de déplacement. Dans le cas de la voiture particulière, la demande s'accroît lorsque le coût d'usage de la voiture se réduit. Elle s'accroît également lorsque le revenu, le coût et le temps d'attente des transports en commun augmentent. De même, la demande de déplacement en transports en commun s'accroît lorsque le coût d'usage de la voiture augmente et se réduit lorsque le coût et le temps d'attente des transports en commun augmentent. 
L’élasticité de la demande au prix du carburant permet d'approfondir la connaissance de cet effet prix. La hausse du prix des carburants limite ainsi la mobilité en voiture (Goodwin, 1992). Toutefois, la valeur des coefficients fluctue en fonction du modèle, des données utilisées (en coupe transversale, en série temporelle, en panel, à court ou à long termes) et du pays concerné (Hanly et al. 2002 ; Holmgren, 2007). Bresson et al. (2004) montrent également que l'élasticité de la demande au prix des carburants est positive dans le cas des transports en commun. Toutefois, cette élasticité est plus faible que l'élasticité de la demande à la variation du tarif des transports en commun. Cela les conduit à penser qu'une mesure de réduction des tarifs peut jouer un rôle substantiel pour accroître l'utilisation des transports en commun.

\subsection{L'effet revenu}

Une des difficultés de l'investigation sur la variable revenu est que celle-ci est corrélée à des variables socio-démographiques. Parmi ces variables, on trouve la taille du ménage (Lyons et al., 2002) et la situation économique (Gakenheimer, 1999) ${ }^{2}$. Il est même possible d'intégrer l'environnement de la maison - à travers par exemple le fait d'être propriétaire ou locataire ou de détenir une ou plusieurs habitations (Meurs et al., 2001), ou d'autres variables sociétales comme la renaissance de la famille ou de la communauté et le taux de fertilité (Lyons et al., 2002).

Mogridge (1967) utilise une distribution des revenus et des dépenses pour estimer le nombre de voiture dans trente ans. Toutefois, les valeurs qu'il obtient pour l'élasticité au revenu et l'élasticité au prix de la demande de voiture seront critiquées par Evans (1970) car elles ne tiennent pas compte de l'inflation. Dargay et Hanly (2002), de même que Bresson et al. (2004), mettent en évidence une relation négative entre le nombre de déplacement en bus et le niveau de revenu. A l'inverse, ils montrent une relation positive entre le revenu et l'utilisation de la voiture.

La variable de revenu permet également de mettre en évidence des différences entre les pays. S’intéressant aux différents stocks de véhicules et au développement économique de différents pays, Dargay et Gately (1999) montrent que la hausse du taux de motorisation, liée à la hausse du revenu per capita, est d'autant plus forte que l'économie du pays se trouve en situation de croissance économique rapide (comme la Corée du Sud et Taiwan). Button et al. (1993) s'intéressent particulièrement aux pays aux revenus les plus faibles. Ils montrent que le taux de motorisation devient d'autant plus sensible au revenu que ces deux dernières variables s'accroissent. Toutefois, si le stock de véhicules est dépendant du niveau de revenu per capita, le niveau de saturation du marché varie selon les pays (Medlock et Soligo, 2002).

Pour prendre en compte l'effet du revenu sur la mobilité, Schafer et al. (2000) utilisent la notion de Budget Temps Transport (BTT) mise en évidence par Zahavi (1973), Roth et Zahavi (1981). Ils l'appliquent à 11 régions sur des données 1960-1990 et montrent que dans les groupes de pays où les revenus sont les plus élevés, comme l'Amérique du Nord et l'Europe, la mobilité et le revenu ont augmenté dans les mêmes proportions. Si on affine l'analyse, on peut néanmoins trouver des différences significatives entre ces groupes: par exemple à $10000 \$$ per capita, la mobilité per capita des pays d'Europe de l'ouest n'est que de 60\% de celle des pays d'Amérique du Nord. Cet écart reflète des différences en infrastructure, en densité urbaine, en population, en coûts d'usage des modes de transport. Ces différences de comportement de mobilité entre les pays sont également mises en avant par van de Coevering et Schwanen (2006).

\subsection{L'effet quantité}

Mogridge (1967 ; 1989) montre que la demande est aussi impactée par un effet quantité. Celui-ci s’évalue à partir des quantités disponibles de biens et de services, mesurées en nombre de déplacement en voiture, en taux de motorisation (Jansson, 1989) et en nombre de siéges- 
kilomètres offerts par les transports en commun (Bresson et al., 2004). Il apparaît que la croissance économique conduit à une hausse du taux de motorisation (Ortuzar et al., 2006). Plus globalement, l'accroissement de la quantité de bien disponible (voiture ou transports en commun) a un effet positif sur la demande. Pour la période 1975-1995, Bresson et al. (2004) montrent que la baisse de l'usage des transports en commun est principalement due à la hausse de la motorisation ; même si cet effet est de moins en moins important dans le temps depuis que la hausse du stock de voiture se ralentit.

L’analyse de la qualité des transports en commun, mesurée en termes de fréquence et de vitesse et de densité du réseau, fait également l'objet d'investigations (Vande Walle et al., 2006). Les résultats montrent que l'impact de l'amélioration de la qualité des transports en commun sur la hausse de la demande est finalement assez faible (Bresson et al., 2004).

\subsection{L'effet spatial}

La problématique spatiale a été longtemps un sujet relevant essentiellement du domaine de la géographie et des études urbaines (rôle de la forme urbaine sur la croissance économique par exemple) et qui ne concernait pas ceux qui travaillaient sur les transports. Des brèches dans ce cloisonnement disciplinaire sont progressivement apparues.

Côté transport ${ }^{3}$, Dargay et Hanly (2004) mettent en avant la nécessité de prendre en compte la relation entre le transport et l'utilisation de l'espace. Kain et Fauth (1977) intègrent le développement urbain, mesuré par la densité dans chaque zone, ainsi que les caractéristiques socio-économiques des ménages, la localisation de leurs emplois et de leurs résidences, pour expliquer le choix de leur mode de déplacement. Pour Small et Verhoef (2007), le type et la densité des bâtiments ainsi que le type d'activité sont des facteurs qui influencent les décisions de déplacement. Button et al. (1993) montrent une relation positive entre le taux de motorisation et le niveau d'urbanisation. Mais cette relation n'est vraie que jusqu'à un certain point. Au-delà de ce point, la saturation des infrastructures est telle que, plus la densité urbaine est forte, plus l'utilisation de la voiture, le taux de motorisation, le nombre de déplacements et la consommation d'énergie se réduisent (Camagni et al., 2002).

Comme le rappelle Crane (2000), l'impact de l'utilisation de l'espace urbain sur la mobilité reste néanmoins difficile à identifier. Handy (1996) fait une revue des différentes méthodes pour étudier le lien entre la forme urbaine et le comportement de déplacement. Elle montre que la mixité des fonctions a un effet négatif sur l'utilisation de la voiture, tout en soulignant la complexité de ce résultat. Cette complexité se retrouve également sur la question de la morphologie de la ville, même si une structure polycentrique semble être plus économe en énergie consommée dans le trafic. Plus une ville est grande, plus la longueur des trajets s'accroît. Or il semble finalement que la taille de la ville n'ait pas d'effet direct sur le choix du mode de transport.

Le coût d'usage du mode de transport, le revenu et les quantités disponibles de bien et de service sont donc devenues des variables structurelles classiques pour estimer la demande de transport urbain. Les variables spatiales sont également prises en compte désormais dans les estimations. La densité urbaine semble d'ailleurs une variable plus facile à utiliser que la morphologie de la ville ou que la répartition des fonctions urbaines. Par contre, les variables de qualité de service des transports en commun paraissent avoir un effet plus faible sur la variation de la demande. Enfin, des interrogations subsistent quant aux différences éventuelles de mobilité entre les groupes de pays. 


\section{Un exemple d'estimation de la demande de transport urbain}

Après avoir brièvement rappelé le contenu de la base de données utilisée, nous présenterons le modèle de demande de transport retenu et nous expliquerons également la méthode d'estimation.

\subsection{Les données de la base IUTP}

La base de données IUTP contient des données collectées en 1995 pour 100 villes dans le monde. Même si la date de collecte des données est maintenant un peu ancienne et a pu faire l'objet de critiques (reprises par van de Coevering et Schwanen, 2006), elle autorise toutefois des comparaisons internationales sur la démographie (population, ...), la structure urbaine (densité urbaine, ...), l'économie (GDP, ...), le système de transport (longueur des routes, ...) (Bonnafous, 2003). En outre, comme le remarquent à juste titre Joly et al. (2006), l'intégralité des 175 indicateurs est renseignée pour 84 des 100 villes de la base.

Notre objectif, dans cet exercice, est de donner des ordres de grandeur dans une perspective de long terme. Dès lors, il n'est pas critique d'avoir des données récentes, d'autant plus que les comportements changent lentement. Nous avons néanmoins identifié des problèmes d'estimation sur les déplacements à pied et à bicyclette ${ }^{4}$. Nous ferons dès lors le choix de n'étudier que l'automobile et les transports en commun.

\subsection{La fonction de demande}

En suivant les résultats de la revue de la littérature, nous choisissons la forme générale de la fonction de demande de déplacement, ou mobilité, notée $D$, suivante:

$D=f\left(p_{V P}, p_{T C}, q_{V P}, q_{T C}, R, s_{U}\right)$

Avec $\mathrm{p}_{\mathrm{vp}}$ : coût de la voiture particulière, $\mathrm{p}_{\mathrm{tc}}$ : coût des transports en commun, $\mathrm{q}_{\mathrm{vp}}$ : quantité de voiture offerte, $\mathrm{q}_{\mathrm{tc}}$ : quantité offerte de transports en commun, $\mathrm{R}$ : revenu, $\mathrm{s}_{\mathrm{u}}$ : la structure urbaine.

Elle se décompose en une mobilité en voiture, notée $D_{C A R}$, évaluée par les déplacements quotidiens en voiture par personne et une mobilité en transports en commun, notée $D_{P T}$, évaluée par les déplacements quotidiens en transports en commun par personne :

$D_{C A R}=f(C C A R, C P T, L R O A D P S U R F, G D P, D E N S)$

$D_{P T}=f(C C A R, C P T, P T V E H S, G D P, D E N S)$

Les variables explicatives se décomposent de la manière suivante:

- le coût de la voiture ( $\mathrm{p}_{\mathrm{VP}}$ ) s'obtient par le coût d'usage moyen pour un déplacement en voiture - noté CCAR -; le coût des transports en commun ( $\mathrm{p}_{\mathrm{TC}}$ ) s'obtient par le coût d'usage moyen pour un déplacement en transports en commun - noté CPT,

- les quantités offertes (qvp, $\mathrm{q}_{\mathrm{TC}}$ ) s’obtiennent respectivement par la longueur des routes pour 1000 habitants rapportée à la surface urbaine - notée LROADPSURF - et le nombre de véhicule kilomètre de service en transports en commun par personne rapporté à la surface urbaine - noté PTVEHS, 
- le revenu (R) est estimé par le PIB urbain par personne - noté GDP,

- la structure urbaine $\left(\mathrm{s}_{\mathrm{u}}\right)$ est obtenue grâce à la densité urbaine - notée DENS.

A défaut de variable de revenu, indisponible dans la base, le $\mathrm{PIB}^{5}$ urbain nous donne une indication du niveau de vie dans la ville. Par ailleurs, l'absence de données en Parité de Pouvoir d'Achat indique un biais important dans la comparaison des villes. En effet, les différences de niveaux de prix et de variation des taux de change ne sont pas neutralisées. Ce biais peut expliquer pourquoi les données recueillies sont parfois surprenantes. Par exemple en 1995, le PIB de Paris serait quasiment le double de celui de Londres (41 000 \$ contre $22300 \$)^{6}$.

Enfin, nous faisons l'hypothèse que la demande estimée est satisfaite par l'offre disponible.

\subsection{Méthode d'estimation}

En faisant apparaître les coefficients de la droite de régression comme des coefficients d'élasticité, nous estimons une relation log-log entre la demande de déplacement (en voiture et en transports en commun) et les variables explicatives de prix, de quantité, de revenu et de structure urbaine. Cette transformation a également l'intérêt de réduire les risques d’hétéroscédasticité.

Pour estimer les paramètres inconnus du modèle, nous utilisons d'abord la méthode d'estimation par les Moindre Carrés Ordinaires (OLS). Puis nous utilisons les tests de BreuschPagan et $t \mathrm{HC}^{7}$ pour traiter le problème d'hétéroscédasticité du modèle. La méthode des Doubles Moindre Carrés (2SLS) nous permet également d'éviter le risque d'autocorrélation des erreurs ${ }^{8}$ entre les variables explicatives.

Comme nous avons choisi de décomposer la demande de déplacement en un modèle de la mobilité en voiture et en un modèle de la mobilité en transports en commun, nous étudions ensuite la relation entre ces deux modèles. Avec la méthode SUR (Seemingly Unrelated Regression) qui teste l'autocorrélation des résidus entre les deux équations, nous vérifions si d'éventuelles variables indépendantes n'auraient pas été omises parce qu'elles seraient incluses dans les résidus. L'utilisation de cette technique ne changeant pas le niveau des estimateurs, nous poursuivons en utilisant la méthode des Triples Moindre Carrés (3SLS) qui s’appuie sur les deux méthodes précédentes, 2SLS et SUR. Il est important de noter que l'utilisation de ces techniques ne conduit pas à des changements importants des coefficients estimés par la méthode OLS.

Des différences de comportements de mobilité semblent exister entre les pays. Nous allons donc vérifier si la création de groupes de mobilité rend nos modèles instables en confrontant notre modèle de référence à d'autres modèles. Le modèle de référence est construit sur l'ensemble des pays de la base de données. Les autres sont construits sur des sous-groupes de pays. Nous utilisons ensuite un test de Chow pour tester la stabilité des modèles. Cet exercice a cependant des limites: la faiblesse de l'échantillon de chacun des sous-groupes (une vingtaine de pays) et l'abandon de la variable de PIB urbain.

Nous pouvons néanmoins identifier:

- «Un groupe Nord Américain » qui bénéficie d’un haut niveau de développement économique, connaît une faible densité urbaine et une domination des déplacements en voiture,

- «Un groupe Asie Développée » avec un haut niveau de développement économique, une forte densité urbaine et une forte utilisation des transports en commun, 
- «Un groupe Européen » qui bénéficie d’un haut niveau de développement économique et qui se positionne entre les situations des deux groupes, Nord Américain et Asie Développée, pour la densité urbaine et le partage modal,

- « Un groupe Autre Pays » sans caractéristiques communes identifiables et qui regroupe les autres pays.

\section{-4 -}

\section{Résultats de l'estimation et application au cas chinois}

Nous allons, dans un premier temps, analyser les résultats des modèles d'estimation de la mobilité en voiture et en transports en commun. Nous les appliquerons ensuite au cas chinois en 2050.

\subsection{Résultats de l'estimation et commentaires}

\subsubsection{La demande de mobilité en voiture}

Pour la mobilité en voiture, les résultats de la régression par la méthode OLS montrent que plus une ville est riche, avec une offre en infrastructures routières importante, plus la mobilité en voiture est forte. A l'inverse, celle-ci apparaît fortement contrainte par une densité urbaine et un coût d'usage moyen des déplacements en voiture élevés (Annexe 1).

Par ailleurs, et contrairement aux travaux initiaux de Mc Fadden, la mobilité en voiture particulière semble décroître lorsque le coût moyen d'un déplacement en transports en commun augmente. Comme la demande de mobilité est sensible à la variation des prix, cette corrélation négative peut s'expliquer par une assimilation de la variation du prix des transports en commun à celle de l'ensemble des prix des transports. L'existence d'une corrélation positive entre le coût d'usage moyen d'un déplacement en voiture et le coût d'usage d'un déplacement en transports en commun (valeur estimée du coefficient : + O,5305, t-value : 5,143) conforte cette explication.

Le modèle de mobilité en voiture qui résiste au problème d'hétéroscédasticité ne contient que deux des cinq variables structurelles : le coût d'usage moyen d'un déplacement en voiture et la densité urbaine (Tableau 1). Sans surprise, les résultats montrent que la mobilité en voiture est d'autant plus élevée que le coût d'usage moyen d'un déplacement en voiture et que la densité urbaine sont faibles. La valeur obtenue pour l'élasticité de la demande de voiture au coût d'usage de la voiture est cohérente avec celle obtenue par Mc Fadden en 1974 (selon le modèle qu'il utilise, il obtient une valeur comprise entre -0,32 et -0,47 - voir Mc Fadden p. 325 et 327).

La méthode 2SLS nous permet d'isoler les effets propres de chacune de ces deux variables et d'éviter ainsi tout problème d'autocorrélation. Les résultats obtenus sont significatifs mais modifient l'estimation des coefficients : l'effet du coût d'usage moyen d'un déplacement en voiture diminue au profit de celui de la densité urbaine (Tableau 1). Le résultat obtenu pour la densité urbaine se situe dans une fourchette allant de $-1,18$ à - 0,25 , ce qui est cohérent avec les résultats des travaux de Giuliano et Dargay (2006, p.118).

Tableau 1 : Résultats des différentes méthodes d’estimation pour le modèle de mobilité en voiture

\begin{tabular}{|c|c|c|c|c|c|c|}
\hline & \multicolumn{2}{|c|}{ Estimation OLS (1) } & \multicolumn{2}{|c|}{ Estimation 2SLS (2) } & \multicolumn{2}{|c|}{ Estimation 3SLS (3) } \\
\hline & Log (CCAR) & Log (DENS) & $\begin{array}{c}\text { Log } \\
\text { (CCAR) }\end{array}$ & Log (DENS) & $\log ($ CCAR) & Log (DENS) \\
\hline Estimate & -0.52185 & -0.39080 & -0.8623 & -0.2300 & -0.521847 & -0.390797 \\
\hline Std. Error & 0.04920 & 0.04495 & 0.2132 & 0.1118 & 0.049196 & 0.044947 \\
\hline
\end{tabular}




\begin{tabular}{|c|c|c|c|c|c|}
\hline t value & -10.607 & -4.045 & -2.057 & -10.60744 & -8.69459 \\
\hline $\operatorname{Pr}(>|t|)$ & 3.19e-13 *** & $1.189^{\mathrm{e}}-04$ & $4.292 \mathrm{e}-02$ & $0 * * *$ & $0 * * *$ \\
\hline $\begin{array}{c}\text { THC } \\
\text { (intercept) }\end{array}$ & -9.772747 & - & - & - & . \\
\hline Estimate & 4.51598 & 5.6756 & & 4.51598 & \\
\hline Std. Error & 0.22816 & 0.7520 & & 0.228164 & \\
\hline T value & 19.793 & 7.547 & & 19.792655 & \\
\hline $\operatorname{Pr}(>|t|)$ & $<2 e-16 * * *$ & $5.813 e-11$ & & $0 * * *$ & \\
\hline THC & 15.879866 & - & & - & \\
\hline Breusch-Pagan test & $\mathrm{BP}=19.2, \mathrm{p}$-value $=6.827 \mathrm{e}-05, \mathrm{df}=2$ & & & & \\
\hline Multiple R square & 0.8269 & & & 0.826908 & \\
\hline Adjusted R square & 0.8226 & & & 0.822634 & \\
\hline
\end{tabular}

\subsubsection{La demande de mobilité en transports en commun}

Une régression de la demande de déplacement en transports en commun par la méthode OLS met en évidence le point suivant : si la mobilité en transports en commun est favorisée par la hausse du coût d'usage moyen d'un déplacement en voiture, la densité urbaine ou l'offre de service de transports en commun par personne, elle est au contraire contrainte par la hausse du coût d'usage des transports en commun et la baisse du PIB urbain (Annexe 2).

Un test de Student plus approfondi (tHC) montre que, contrairement à ce que l'on pourrait attendre, le PIB urbain n'est pas significativement corrélé à la mobilité en transports en commun. Ce résultat confirme nos doutes sur la construction de cette variable dans la base de données IUTP et nous conduit à l'exclure du modèle. L'utilisation du PIB urbain en Parité de Pouvoir d'Achat apparaît être un meilleur indicateur du niveau de richesse d'une agglomération. Il fixe, en effet, un référentiel commun qui, en éliminant les différences de niveaux de prix et de variation de taux de change entre les villes, permet de mieux les comparer.

Une fois le PIB urbain exclu, le modèle qui résiste, corrèle la mobilité en transports en commun aux trois variables suivantes : la hausse du coût d'usage moyen pour un déplacement en voiture, la densité urbaine et la baisse du coût d'usage des transports en commun (Tableau 2).

Les résultats de la méthode 2SLS sont toujours significatifs mais d'importantes modifications sur les niveaux des coefficients apparaissent (Tableau 2). Ces nouveaux coefficients d'élasticité accentuent le poids des variables du coût d'usage de la voiture et des transports en commun et réduisent celui de la densité urbaine. La valeur du coefficient obtenue pour l'élasticité de la demande pour les transports en commun au coût d'usage de la voiture $(+0,91)$ devient ainsi presque identique à celle qu'avait identifiée Mc Fadden en 1974 (selon le modèle utilisé, il trouve une valeur comprise entre +0,81 et +0,97 - voir Mc Fadden p. 325 et 327). Par ailleurs, Bresson et al. (2004, p.274) estiment l'élasticité minimale à long terme des transports en commun à leurs tarifs ${ }^{9}$ à $-1,56$. Avec une élasticité de la mobilité en transports en commun au coût des transports en commun de $-1,7$, nous n'en sommes pas très loin.

Dans cette estimation, l'action combinée du coût d'usage de la voiture particulière et de la densité urbaine ne suffisent pas à compenser l'effet négatif d'une hausse du coût d'usage des transports en commun sur la mobilité en transports en commun.

Tableau 2 : Résultats des différentes estimations pour le modèle de mobilité en transports en commun

\begin{tabular}{c|ccc|ccc|ccc}
\hline & \multicolumn{3}{|c|}{ Estimation OLS (1) } & \multicolumn{3}{c|}{ Estimation 2SLS (2) } & \multicolumn{3}{c}{ Estimation 3SLS (3) } \\
\cline { 2 - 10 } & $\begin{array}{c}\log \\
\text { (CCAR) }\end{array}$ & $\log ($ CPT) & $\begin{array}{c}\text { Log } \\
\text { (DENS) }\end{array}$ & $\begin{array}{c}\text { Log } \\
\text { (CCAR) }\end{array}$ & $\begin{array}{c}\text { Log } \\
\text { (CPT) }\end{array}$ & Log (DENS) & Log (CCAR) & Log (CPT) & Log (DENS) \\
\hline Estimate & 0.35296 & -0.52756 & 0.43146 & 0.9118 & -1.7030 & 0.3989 & 0.363881 & -0.550524 & 0.430821
\end{tabular}




\begin{tabular}{|c|c|c|c|c|c|c|c|c|c|}
\hline Std. Error & 0.11681 & 0.11040 & 0.09539 & 0.3727 & 0.7057 & 0.1495 & 0.115426 & 0.103772 & 0.095381 \\
\hline t value & 3.022 & -4.779 & 4.523 & 2.4466 & -2.4131 & 2.6683 & 3.152505 & -5.305133 & 4.51684 \\
\hline $\operatorname{Pr}(>|t|)$ & $\begin{array}{c}0.00337 \\
* *\end{array}$ & $\begin{array}{c}7.88 \mathrm{e}-06 \\
* * *\end{array}$ & $\begin{array}{c}2.09 \mathrm{e}-05 \\
* * *\end{array}$ & 0.016614 & 0.018102 & 0.009227 & $0.002279 * *$ & $1 \mathrm{e}-06 * * *$ & $2.1 \mathrm{e}-05 * * *$ \\
\hline $\begin{array}{c}\mathrm{THC} \\
\text { (intercept) }\end{array}$ & 1,709982 & $-2,528109$ & 2,638667 & - & - & - & - & - & - \\
\hline Estimate & -2.58959 & & & -1.0709 & & & -2.559916 & & \\
\hline Std. Error & 0.50454 & & & 1.1821 & & & 0.502191 & & \\
\hline t value & -5.133 & & & -0.9059 & & & -5.097497 & & \\
\hline $\operatorname{Pr}(>|t|)$ & $1.96 \mathrm{e}-06$ ** & & & 0.367705 & & & $2^{\mathrm{e}}-06 * * *$ & & \\
\hline $\mathrm{THC}$ & $-3,993859$ & & & - & & & - & & \\
\hline $\begin{array}{l}\text { Breusch- } \\
\text { Pagan test }\end{array}$ & $\mathrm{BP}=10.52$ & 68 , p-value $=$ & $=0.01458$, & $\mathrm{Df}=3$ & & & & & \\
\hline $\begin{array}{c}\text { Multiple R } \\
\text { Squared }\end{array}$ & 0.4306 & & & & & & 0.430252 & & \\
\hline $\begin{array}{c}\text { Adjusted R } \\
\text { squared }\end{array}$ & 0.4092 & & & & & & 0.408886 & & \\
\hline
\end{tabular}

\subsubsection{Relation entre les deux modèles de demande}

Les résultats obtenus avec les méthodes SUR et 3SLS (Tableau 1, Tableau 2) ne modifient pas la significativité des résultats ; les nouveaux coefficients sont quasiment similaires à ceux de l'estimation par la méthode OLS. Dès lors, et pour la suite de ce papier, nous n’utiliserons que les résultats donnés par l'estimation OLS.

\subsubsection{Stabilité des modèles de demande}

Le test de Chow est significatif pour les deux modèles: nous ne rejetons donc pas l'hypothèse $\mathrm{H}_{0}$ de stabilité des modèles de demande (Tableau 3). Cette stabilité des deux modèles démontre que les variables qui agissent sur la mobilité sont indépendantes du groupe de pays d'appartenance.

Ce résultat s'explique principalement par le fait que nous avons choisi d'exclure la variable de PIB urbain. Or, ce sont le plus souvent les différences de revenu ou de développement entre les pays qui expliquent les différences de mobilité.

Tableau 3 : Résultats du test de Chow pour les deux modèles

\begin{tabular}{c|cccc|ccc}
\hline & \multicolumn{3}{|c|}{ Modèle de la mobilité en voiture (1) } & \multicolumn{3}{c}{ Modèle de la mobilité en transports en } \\
commun (2)
\end{tabular}


Signif. codes: $0^{\prime * * * *^{\prime}} 0.001^{\prime * * \prime} 0.01^{\prime *}{ }^{\prime *} 0.05^{\prime}$ '. $0.1^{\prime}$ ' $^{\prime} 1$ (1) Residual standard error: 0.3071 on 78 degrees of freedom, Multiple R-Squared: 0.8532, adjusted R-squared: 0.8419 , F-statistic: 75.57 on 6 and $78 \mathrm{DF}$, p-value: $<2.2 e-16$, Car mobility model formula log (CCAR) + log $(D E N S)+$ groupe - 1 (2) Residual standard error: 0.5828 on 77 degrees of freedom, Multiple R-Squared: 0.8163, Adjusted R-squared: 0.7996, F-statistic: 48.89 on 7 and $77 \mathrm{DF}$, p-value: <2.2e-16, Formula for the public transport mobility model log $(C C A R)+\log (D E N S)+\log (C P T)$ + groupe -1

\subsection{Une application au cas chinois en 2050}

\subsubsection{Méthode de construction des scénarios}

L'exercice de projection dans l'avenir est un exercice périlleux par nature. C'est pourquoi nous allons l'aborder sous forme de scénarios, présentant des ordres de grandeurs de futurs possibles.

New York, Hong Kong, Paris seront nos villes de référence (Tableau 4): en faisant l'hypothèse qu'en 2050, Pékin ressemblera au Hong Kong d'aujourd'hui (même densité urbaine par exemple), nous pouvons estimer sa mobilité grâce aux équations de demande précédemment calculées.

New York représente aujourd'hui l'une des plus grandes villes Nord américaines: niveau de développement élevé, densité urbaine faible, important étalement urbain, domination de la voiture favorisée par un coût d'usage moyen faible. Hong Kong est une ville asiatique très développée, dense et compacte, à forte densité urbaine. La part modale des transports en commun y est très élevée et leur coût d'utilisation se situe dans la moyenne des grandes villes du monde. Enfin, Paris se situe entre ces deux villes pour sa densité urbaine et son partage modal.

Nous allons à présent appliquer les deux modèles de demande aux trois plus grandes villes chinoises que sont Pékin, Shanghai et Guangzhou. Pour caler notre estimation, nous utilisons les données disponibles dans la base IUTP pour ces trois villes. Ces villes sont aujourd'hui suffisamment différentes pour que nous obtenions des résultats contrastés: Pékin et Guangzhou ont une densité urbaine élevée mais inférieure de moitié à celle de Shanghai. Malgré tout, Shanghai reste encore loin du niveau explosif de Hong Kong. Si l'on regarde le coût d'usage pour un déplacement en transports en commun, les différences sont également importantes entre ces trois villes : à Guangzhou et à Shanghai le coût d'usage est respectivement trois fois et deux fois plus élevé qu'à Pékin. A l'inverse, le coût d'usage d'un déplacement en voiture est deux fois plus élevé à Pékin qu’à Shanghai ou Guangzhou (Tableau 4).

Il faut toutefois se garder de généraliser ces résultats à toutes les villes chinoises. Ces trois grandes villes ne sont pas forcément représentatives de la situation dans le reste du pays (Brun et al., 2002).

Les données des villes de référence sont toujours celles de la base IUTP et sont présentées ci-dessous.

Tableau 4 : Valeur des variables explicatives pour les villes de référence des scénarios

\begin{tabular}{c|c|c|c}
\hline Villes & Densité urbaine & $\begin{array}{c}\text { Coût d'usage moyen } \\
\text { pour un déplacement } \\
\text { en voiture }\end{array}$ & $\begin{array}{c}\text { Coût d'usage moyen } \\
\text { pour un déplacement } \\
\text { en transports en } \\
\text { commun }\end{array}$ \\
\hline New York & 18 & 83 & 39 \\
Hong Kong & 320 & 220 & 29 \\
Pékin & 123 & 547 & 15 \\
Shanghai & 196 & 274 & 31 \\
Guangzhou & 119 & 267 & 58 \\
Paris & 48 & 123 & 27 \\
\hline
\end{tabular}


Les unités sont les suivantes : la densité urbaine est en ha/personne, les coûts d'usage moyen pour un déplacement quel que soit le mode sont exprimés en pourcentage du GDP/déplacement et par personne.

\subsubsection{Lecture des résultats}

Nous pouvons lire nos résultats comme des résultats de variation.

En effet, en partant des équations de demande estimées:

$$
\begin{aligned}
& \log \left(D_{C A R}\right)=\alpha_{1} \log (C C A R)+\alpha_{2} \log (D E N S)+c \\
& \log \left(D_{P T}\right)=\alpha_{1} \log (C C A R)+\alpha_{2} \log (C P T)+\alpha_{3} \log (\text { DENS })+c
\end{aligned}
$$

Nous en déduisons les expressions :

$$
\begin{aligned}
& \frac{d D_{C A R}}{D_{C A R}}=\alpha_{1} \frac{d C C A R}{C C A R}+\alpha_{2} \frac{d D E N S}{D E N S} \\
& \frac{d D_{P T}}{D_{P T}}=\alpha_{1} \frac{d C C A R}{C C A R}+\alpha_{2} \frac{d C P T}{C P T}+\alpha_{3} \frac{d D E N S}{D E N S}
\end{aligned}
$$

Nous pouvons ensuite estimer la situation future de Pékin, Shanghai et Guangzhou en multipliant cette élasticité par la valeur des variables explicatives significatives (CCAR, DENS et CPT) pour ces trois villes en 1995.

\subsubsection{Principaux résultats}

La croissance économique et l'exode rural de ces dernières années ont induit une forte hausse de la population urbaine chinoise (Bouf, 2006 ; Deng et al., 2008). Zhang (2002) montre que le GDP per capita a un effet positif sur la croissance de la population des villes chinoises, qu'elles soient ou non situées sur la côte (Chen et Coulson, 2002). Ces villes se caractérisent par une forte densité humaine dans l'occupation de l'espace (Zhou et Ma, 2000). Il paraît donc très improbable qu'en 2050, Pékin, Shanghai ou Guangzhou se rapprochent de la faible densité urbaine de New York ou de celle plus moyenne de Paris (Tableau 4).

Par ailleurs, nous allons établir deux scénarios différents pour chacune de ces trois villes : un " scénario haut » où la ville de comparaison est Hong Kong aujourd'hui et un "scénario médian » où la ville de référence est Shanghai aujourd’hui.

Les estimations (Tableau 5) montrent qu'en 2050, si Pékin subissait une pression de la densité urbaine aussi forte que Hong Kong aujourd'hui, moins de 50\% des déplacements seraient réalisés en voiture. Ce pourcentage s'obtient quel que soit le coût moyen de ce déplacement.

Pour Shanghai et Guangzhou, ce chiffre serait de moins de 30\% (entre 16 et 27\%).

En 2050, si Pékin et Guangzhou avaient une densité urbaine équivalente à celle de Shanghai aujourd'hui, 60-70\% des déplacements à Pékin se feraient en voiture contre 30-40\% à Guangzhou. Ces pourcentages correspondent respectivement à la situation que connaissent aujourd'hui Paris (72\% des déplacements quotidiens en voiture) et Shanghai (31\% des déplacements quotidiens en voiture) (Tableau 5).

Même si des différences existent, les scénarios montrent que les perspectives du marché de l'automobile en Chine sont bonnes.

Tableau 5 : Estimations des déplacements quotidiens en voiture à Pékin, Shanghai et Guangzhou en 2050 


\begin{tabular}{c|ccc} 
& Hong Kong & Paris & New York \\
\hline Si Pékin avait la densité urbaine de Hong Kong & $\mathbf{0 , 4 0}$ & $\mathbf{0 , 4 6}$ & $\mathbf{0 , 4 8}$ \\
Si Pékin avait la densité urbaine de Shanghai & $\mathbf{0 , 6 4}$ & $\mathbf{0 , 6 9}$ & $\mathbf{0 , 7 1}$ \\
\hline Si Guangzhou avait la densité urbaine de Shanghai & $\mathbf{0 , 3 2}$ & $\mathbf{0 , 3 9}$ & $\mathbf{0 , 4 2}$ \\
Si Guangzhou avait la densité urbaine de Hong Kong & $\mathbf{0 , 1 6}$ & $\mathbf{0 , 2 4}$ & $\mathbf{0 , 2 7}$ \\
\hline Si Shanghai avait la densité urbaine de Hong Kong & $\mathbf{0 , 1 9}$ & $\mathbf{0 , 2 3}$ & $\mathbf{0 , 2 5}$ \\
\hline
\end{tabular}

Les résultats des scénarios sur la mobilité quotidienne en transports en commun sont plus contrastés (Tableau 6). A Pékin, la mobilité en transports en commun passe d'environ $15 \%$ à $70 \%$ des déplacements selon le scénario retenu. Pour obtenir ce dernier résultat, il faudrait néanmoins que la densité urbaine soit aussi explosive qu’à Hong Kong aujourd’hui et que le coût d'usage des transports en commun soit faible.

Les résultats de Shanghai et Guangzhou sont moins contrastés. Dans tous les scénarios, c'est-à-dire quel que soit le coût d'usage de la voiture et des transports en commun ou la densité urbaine, la mobilité en transports en commun se situe au-delà des 40\%.

Néanmoins, en 2050 même si Shanghai atteignait la densité urbaine de Hong Kong aujourd'hui, elle ne parviendrait pas à égaler la mobilité en transports en commun actuelle de Hong Kong (71\%). En 2050, Shanghai se rapprocherait plutôt de la situation actuelle de Tokyo où 59\% des déplacements s'effectuent en transports en commun.

Tous ces résultats mettent en avant la nécessité d'améliorer l’offre en transports en commun et soulèvent la question du financement des investissements nécessaires.

Tableau 6: Estimations des déplacements quotidiens en transports en commun à Pékin, Shanghai et Guangzhou en 2050

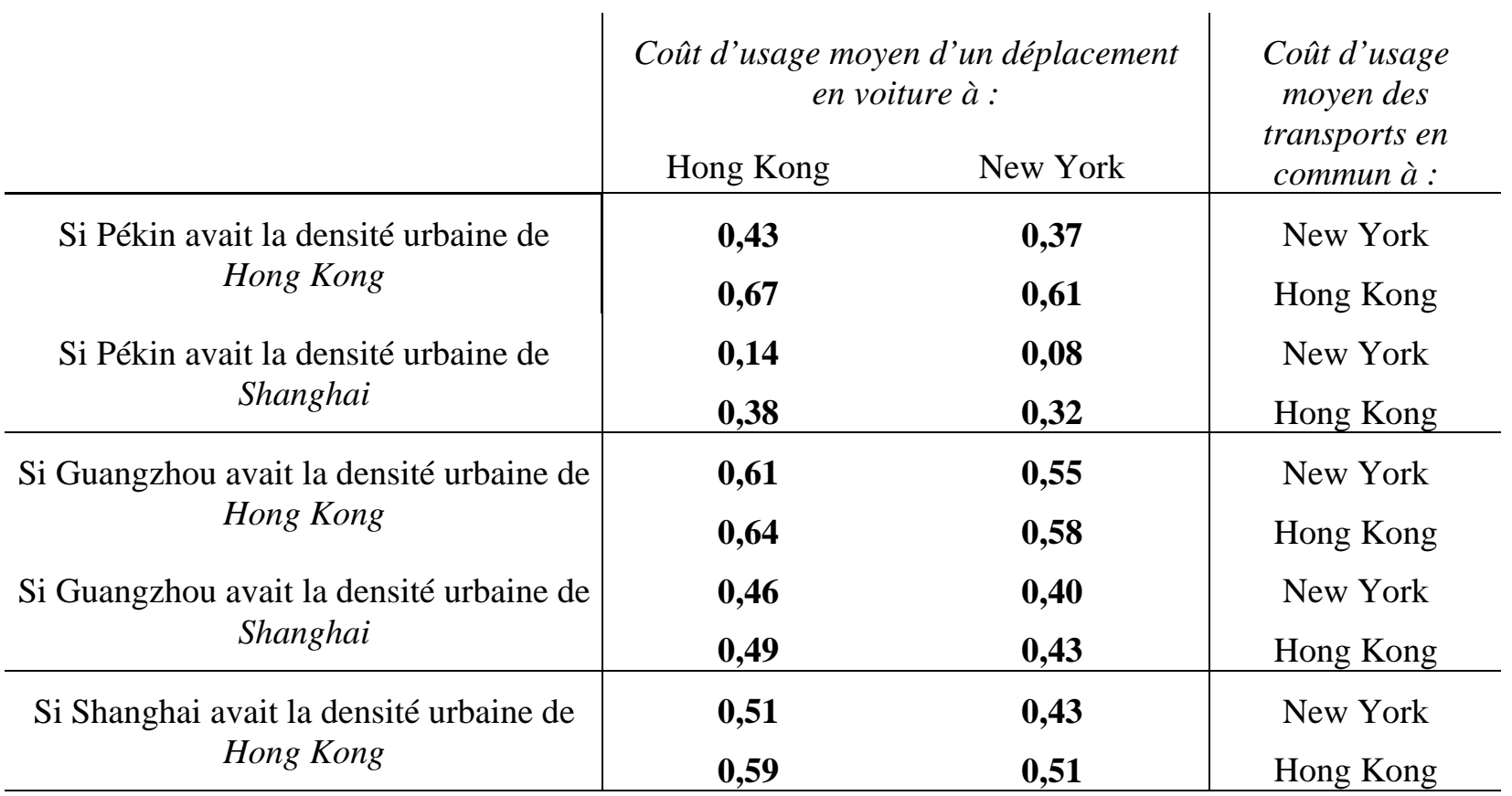




\section{- $5-$ \\ Conclusion}

Pour nous aider à dessiner la ville de demain, nous avons estimé un modèle de demande de transport urbain.

Nos résultats montrent que seules les variables de coût du mode de transport et de densité urbaine sont statistiquement significatives. La mobilité urbaine en voiture s'accroît lorsque le coût d'usage moyen de la voiture et la densité urbaine baissent. A l'inverse, la hausse de ces deux variables associée à la baisse du coût d'usage moyen des transports en commun favorise la mobilité en transports en commun. Ces résultats plaident pour une analyse conjointe des transports et de l'espace dans les travaux d'estimation de la mobilité.

Nos résultats ne nous permettent cependant pas de valider l'hypothèse d'une différence de mobilité basée sur les groupes de pays. Ils soulignent néanmoins la nécessité d'une bonne évaluation de l'effet revenu pour saisir au mieux ces disparités.

Les résultats de l'application au cas chinois montrent sans surprise que les perspectives du marché automobile sont fortes ; plus fortes d'ailleurs à Pékin qu'à Shanghai ou Guangzhou. Dans ces deux dernières villes, la hausse de la densité urbaine combinée à un coût d'usage élevé semble permettre de contenir un peu mieux cette emprise de l'automobile. Ce résultat induit deux conséquences. Il pousse, d'une part, à encourager la poursuite de l'exode rural et incite à une réflexion sur la taille optimale des villes chinoises. Il conduit, d'autre part, à anticiper dès aujourd'hui les effets négatifs de l'augmentation de l'usage de la voiture que sont la congestion et la pollution. Une solution possible consisterait à augmenter les taxes sur les véhicules, soit par le stationnement, soit par la mise en place d'un péage urbain (Raux et Souche, 2004). Cette solution aurait d'ailleurs le double avantage de réduire la pollution et de proposer de nouvelles recettes pour financer les transports en commun, dont nous avons également montré les fortes perspectives de croissance.

\section{Bibliographie}

ANAS, A., ARNOTT, R., SMALL, K.A. (1998) Urban spatial structure, Journal of Economic Litterature, vol 36, p.1426-1464.

BONNAFOUS (DIR), (2003) Une analyse de la base UITP sur les systèmes de transports urbains de 100 villes du monde, Commissariat Général du Plan, 189p.

BOUF, D. (DIR), (2006) Transports en Chine en 2050, Rapport Final, D.A.E.I., janv.

BOYCE, D. (2006) Forecasting travel for very large cities: challenges and opportunities for China, $53^{\text {rd }}$ Annual North Amercian Meetings of Regional Science Association International, Toronto, 16-18 nov.

BRESSON, G., DARGAY, J., MADRE, J-L., PIROTTE, A. (2003) The main determinant of the demand for public transport: a comparative analysis of England and France using shrinkage estimators, Transportation Research Part A, vol 37, p.605-627. 
BRESSON, G., DARGAY, J., MADRE, J-L., PIROTTE, A. (2004) Economic and structural determinants of the demand for public transport : an analysis on a panel of French urban areas using shrinkage estimators, Transportation Research Part A, vol 38, p.269-285.

BRUN, J.F. COMBES, J.L. RENARD, M.F. (2002) Are there spillover effects between coastal and noncoastal regions in China ? China Economic Review, Vol 13, 2-3, p.161-169.

BUTTON, K., NGOE, N., HINE, J. (1993) Modelling vehicle ownership and use in low income countries, Journal of Transport Economics and Policy, vol 27, 1, p.51-67.

CAMAGNI, R., GIBELLI, M-C., RIGAMONTI, P. (2002) Urban mobility and urban form : the social and environmental costs of different patterns of urban expansion, Ecological Economics, vol 40, p.199-216.

CAMERON, I., KENWORTHY, J.R., LYONS, T.J. (2003) Understanding and predicting private motorised urban mobility, Transportation Research Part D, vol 8, p.267-283.

C.E.P.I.I. (2002) Compétitivité des indistries françaises. Les enseignements d'une comparaison européenne, La Lettre du Cepii, n²10, 4p.

CERVERO, R. (1996) Mixed land-uses and commuting : evidence from american housing survey, Transportation Research A, vol 30, 5, p.361-377.

CERVERO, R., WU, K.L. (1998), Sub-centring and commuting : evidence from the San Francisco bay Area, Urban Studies, vol 35, 7, p.1059-1076.

CHEN, A., COULSON, N.E. (2002) Determinant of urban migration : evidence from Chinese cities, Urban Studies, vol 39, 12, p.2189-2197.

CRANE, R. (2000) The influence of urban form on travel : an interpretative review, Journal of Planning Literature, vol 15, 1, p.3-23.

DAEI-SES (2004) La demande de transports en 2025, Ministère des Transports, Chapitre 1.

DARGAY, J., HANLY, M. (2002) The demand for local bus services in England, Journal of Transport Economics and Policy, vol 36, 1, p.73-91.

DARGAY, J., HANLY, M. (2004) Land use and mobility, World Conference on Transport Research, Istanbul.

DARGAY, J., GATELY, D. (1999) Income's effect on car and vehicle ownership, worldwide : 1960-2015, Transportation Research Part A, vol 33, p.101-138.

DAVIDSON, W., DONNELLY, R., VOVSHA, P., FREEDMAN, J., RUEGG, S., HICKS, J., CASTIGLIONE, J., PICADO, R. (2007) Synthesis of first practices and operational research approach in activity-based travel demand modeling, Transportation Research Part A, vol 41, p.464-488.

DENG, X., HUANG, J., ROZELLE, S., UCHIDA, E. (2008) Growth, population, and urban land expansion of China, Journal of Urban Economics, vol 63, p.96-115.

EVANS, A. (1970) The prediction of car ownership. A comment, Journal of Transport Economics and Policy, vol 4, 1, p.89-106.

GAKENHEIMER, R. (1999) Urban mobility in the developing world, Transportation Reasearch Part A, vol 33, p.671-689.

GIULIANO, G., DARGAY, J. (2006) Car ownership, travel and land use : a comparison of the US and Great Britain, Transportation Research Part A, vol 40, p.106-124. 
GOODWIN, P. (1992) A review of new demand elasticites with special reference to short and long run effects of price changes, Journal of Transport Economics and Policy, vol 26, 2, p.155169.

HANDY, S. (1996) Methodologies for exploring the link between urban form and travel behavior, Transportation Research Part D, vol 1, 2, p.151-165.

HANLY, M., DARGAY, J., GOODWIN, P. (2002) Review of income and price elasticities in the demand for road traffic, ESRC TSU publication 2002/13, University of London, 95p.

HOLMGREN, J. (2007) Meta-analysis of public transport demand, Transportation Research Part A, vol 41, p.1021-1035.

HUENEMANN, R. (2001) Are China's recent transport statistics plausible ?, China Economic Review, 12, p.368-371.

I.U.T.P. (2001) Millennium Cities Database for Sustainable Transport.

JANSSON, J.O. (1989) Car demand modelling and forecasting. A new Approach, Journal of transport Economics and Policy, vol 23, 2, p.125-140.

JOLY, I., MASSON, S., PETIOT, R. (2006) Les determinants de la demande en transports collectifs urbains : comparaison internationale et analyse économétrique, Cahiers Scientifiques du Transport, vol 50, p.91-120.

KAIN, J.F., FAUTH, G.R. (1977) The effetcs of urban structure on automobile ownership and journey to work mode choices, Transportation Research Record, p.9-17.

KENWORTHY, J.R., LAUBE, F.B. (1999) Patterns of automobile dependence in cities : an international overview of key physical and economic dimensions with some implications for urban policy, Transportation Research Part A, vol 33, p.691-723.

LYONS, G., CHATTERJEE, K., BEECROFT, M., MARSDEN, G. (2002) Determinants of travel demand - exploring the future of society and lifestyles in the UK, Transport Policy, vol 9, p.17-27.

MC FADDEN, D. (1974) The measurement of urban travel demand, Journal of Public Economics, 3, p.303-328.

MEDLOCK, K., SOLIGO, R. (2002) Car ownership and economic development, with forecasts to year 2015, Journal of Transport Economics and Policy, vol 36, 2, p.163-188.

MEURS, H., HAAIJER, R. (2001) Spatial structure and mobility, Transportation Research Part $D$, vol 6, p.429-446.

MOGDRIDGE, M. (1967) The prediction of car ownership, Journal of Transport Economics and Policy, vol 1, 1, p.55-74.

MOGDRIDGE, M. (1989) The prediction of car ownership and use reviseted. The beginning of the end ?, Journal of Transport Economics and Policy, vol 23, 1, p.55-74.

ORTURZAR, J de D., WILLUMSEN, L.G. (2006), Modelling transport, John Wiley and Sons, 3th edition, 497p.

OUM, T.H., WATERS II, W .J., YONG, J.S. (1992) Concepts of price elasticities of transport demand and recent empirical estimates, Journal of Transport Economics and Policy, vol 26, 2, p.139-154. 
RAUX, C., SOUCHE, S. (2004) The acceptability of urban road pricing: A theoretical analysis applied to experience in Lyon, Journal of Transport Economics and Policy, vol 38, 2, p. 191216.

ROTH, G.T., ZAHAVI, Y. (1981) Travel time «budget » in developing countries, Transportation Research Part A, vol 15, 1, p.87-95.

SCHAFER, A., VICTOR, D. (2000) The future mobility of world population, Transportation Research Part A, vol 34, p.171-205.

SMALL, K.A., VERHOEF, E. (2007) The economics of urban transportation, Routledge, 276p. VAN DE COEVERING, P., SCHWANEN, T. (2006) Re-evaluating the impact of urban form on travel patterns in Europe and North-America, Transport Policy, vol 13, p.229-239.

VANDE WALLE, S., STEENBERGHEN, T. (2006) Space and time related determinants of public transport use in trips chains, Transportation Research Part A, vol 40, p.151-162.

WILSON, D., PURUSHOTHAMAN, R. (2003) Dreaming with BRICs: the path to 2050, Global Economic Paper No : 99, in Goldman Sachs ed.

ZAHAVI, Y. (1973) The TT-relationship : a unified approach to transportation planning, Traffic engineering and Control, p.205-212.

ZHANG, K.H. (2002) What explains China's rising urbanization in the Reform Era, Urban Studies, vol 39, 12, p.2301-2315.

ZHOU, Y., MA, L.J.C. (2000) Economic restructuring and suburbanization in China, Urban Geography, vol 21, 3, p.205-236. 
Annexe 1 : Estimation OLS pour la mobilité en voiture

\begin{tabular}{|c|c|c|c|c|c|}
\hline & $\sim \log ($ CCAR $)$ & $\sim \log ($ DENS) & $\sim \log (\mathrm{CPT})$ & $\sim \log (\mathrm{GDP})$ & $\sim \log ($ LROADPSURF) \\
\hline Estimate & -0.74303 & -0.6373 & -0.40721 & 0.45298 & 0.14847 \\
\hline Std. Error & 0.05819 & 0.0591 & 0.09826 & 0.04758 & 0.05577 \\
\hline t value & -12.77 & -10.78 & -4.144 & 9.520 & 2.662 \\
\hline $\operatorname{Pr}(>|t|)$ & $<2 \mathrm{e}-16 * * *$ & $<2 \mathrm{e}-16 * * *$ & $8.25 \mathrm{e}-05 * * *$ & $6.66 \mathrm{e}-15 * * *$ & $0.00934 * *$ \\
\hline \multicolumn{6}{|l|}{ (intercept) } \\
\hline Estimate & 4.14507 & 2.7385 & 1.73620 & -0.96009 & -0.13989 \\
\hline Std. Error & 0.30974 & 0.2379 & 0.36951 & 0.13753 & 0.16119 \\
\hline t value & 13.38 & 11.51 & 4.699 & -6.981 & -0.868 \\
\hline $\operatorname{Pr}(>|t|)$ & $<2^{e}-16 * * *$ & $<2 e-16 * * *$ & $1.04 e-05 * * *$ & $6.98 e-10 * * *$ & 0.38802 \\
\hline $\begin{array}{c}\text { Multiple R } \\
\text { Squared }\end{array}$ & 0.6654 & 0.5865 & 0.1732 & 0.525 & 0.07956 \\
\hline $\begin{array}{l}\text { Adjusted R } \\
\text { squared }\end{array}$ & 0.6613 & 0.5814 & 0.1631 & 0.5192 & 0.06833 \\
\hline
\end{tabular}

Signif. codes: $0^{\prime * * * \prime} 0.001^{\prime * * \prime} 0.01$

Annexe 2 : Estimation OLS pour la mobilité en transports en commun

\begin{tabular}{|c|c|c|c|c|c|}
\hline & $\sim \log ($ CCAR $)$ & $\sim \log (\mathrm{CPT})$ & $\sim \log ($ GDP) & $\sim \log (\mathrm{DENS})$ & $\sim \log (\mathrm{PTVEHS})$ \\
\hline Estimate & 0.3546 & -0.2228 & -0.05216 & 0.49432 & 0.33690 \\
\hline Std. Error & 0.1102 & 0.1232 & 0.08003 & 0.09181 & 0.04247 \\
\hline t value & 3.219 & -1.810 & -0.652 & 5.384 & 7.933 \\
\hline $\operatorname{Pr}(>|t|)$ & $0.00184 * *$ & 0.074 & 0.516382 & $6.78 \mathrm{e}-07 * * *$ & $9.48 \mathrm{e}-12 * * *$ \\
\hline \multicolumn{6}{|l|}{ (intercept) } \\
\hline Estimate & -2.8478 & -0.1610 & -0.84446 & -2.92330 & -0.62409 \\
\hline Std. Error & 0.5863 & 0.4631 & 0.23131 & 0.36954 & 0.08414 \\
\hline t value & -4.857 & -0.348 & -3.651 & -7.911 & -7.417 \\
\hline $\operatorname{Pr}(>|t|)$ & $5.63 e-06 * * *$ & 0.729 & $0.000459 * * *$ & $1.05 e-11 * * *$ & $9.84 e-11 * * *$ \\
\hline $\begin{array}{l}\text { Multiple R } \\
\text { Squared }\end{array}$ & 0.1122 & 0.0384 & 0.005154 & 0.2612 & 0.4342 \\
\hline $\begin{array}{l}\text { Adjusted R } \\
\text { squared }\end{array}$ & 0.1014 & 0.02667 & -0.006979 & 0.2522 & 0.4273 \\
\hline
\end{tabular}

Annexe 3 : Nombre et pourcentage de déplacements en voiture ou en transports en commun pour quelques villes de la base I.U.T.P.

\begin{tabular}{|c|c|c|c|c|}
\hline $\begin{array}{c}\text { Déplacements dans quelques } \\
\text { villes de la base UITP }\end{array}$ & $\begin{array}{c}\text { Déplacement } \\
\text { quotidien par } \\
\text { personne en voiture } \\
\text { quotidien par } \\
\text { quersonne en voiture }\end{array}$ & $\begin{array}{c}\text { Pourcentage de } \\
\text { puotidien en } \\
\text { transports en } \\
\text { commun }\end{array}$ & $\begin{array}{c}\text { Déplacement } \\
\text { déplacement } \\
\text { quotidien par } \\
\text { personne en } \\
\text { transports en } \\
\text { commun }\end{array}$ \\
\hline New York & 2,49 & 89,57 & 0,29 & 10,43 \\
\hline Tokyo & 0,91 & 50,84 & 0,88 & 49,16 \\
\hline Hong Kong & 0,52 & 28,11 & 1,33 & 71,89 \\
\hline Londres & 1,38 & 75,82 & 0,44 & 24,18 \\
\hline Paris & 1,25 & 72,25 & 0,48 & 27,75 \\
\hline Pékin & 0,59 & 46,46 & 0,68 & 53,54 \\
\hline Shanghai & 0,22 & 31,43 & 0,48 & 68,57 \\
\hline Guangzhou & 0,38 & 53,52 & 0,33 & 46,48 \\
\hline
\end{tabular}




\section{Notes}

${ }^{1}$ Même si les propos de ce papier n'engagent que son auteur, celle-ci voudrait remercier Yves Croissant et Dominique Bouf, respectivement professeur à l'Université Lumière Lyon 2 et chercheur au CNRS, pour leurs conseils avisés. L'auteur voudrait également remercier les relecteurs anonymes de ce papier pour leurs critiques et leurs commentaires constructifs. Enfin, elle adresse ses remerciements à S. Le Corvec pour ses précieuses relectures.

${ }^{2}$ Sur cette question voir également Cameron et al. (2003) et Holmgren (2007).

${ }^{3}$ Côté géographie et études urbaines, on s’est par exemple intéressé à l'impact de la forme urbaine et de la nature du polycentrisme (polycentrisme versus monocentrisme) sur la distance des migrations Anas et al., (1998), Cervero (1996), Cervero et Wu (1998).

${ }^{4}$ Aucun habitant de Madrid n'utilise la bicyclette et la part modale de la marche à pied s'élève à 38\% à Paris en 1995 ! Or en 1994, le Ministère Français des Transports estimait que cette dernière s'élevait à 23\% dans l'aire urbaine de Paris (D.A.E.I.-S.E.S., 2004).

${ }^{5}$ En dollar américain 1990 mais pas en Parité de Pouvoir d'Achat. Voir l'explication qu'en donnent Kenworthy et Laube (p.699, 1999) et notre critique dans la suite du papier.

${ }^{6}$ A la suite de sa sortie du Système Monétaire Européen, la Livre Sterling s’était, entre 1992 et 1995, fortement dépréciée par rapport au Deutsch Mark auquel la monnaie française était arrimée. Le niveau des prix britanniques avait baissé de 73\% par rapport au niveau des prix français en 1995 (CEPII, 2002).

${ }^{7}$ Test $t$ de Student cohérent avec l’heteroscedasticité.

${ }^{8}$ Le test de Breusch-Pagan teste si la variance estimée des résidus de la régression est dépendante de la valeur des variables indépendantes. La méthode 2SLS permet d’identifier des variables instrumentales qui ne sont pas corrélées au terme d'erreur mais uniquement avec l'une des variables explicatives du modèle, ce qui permet d'avoir un estimateur sans biais dû au terme d'erreur.

${ }^{9}$ Elasticité pour des données en semi-log pour la France sur 1975-1995. 